\title{
Solvent Dependence of the Rheological Properties in Hydrogel Magnetorheological Plastomer
}

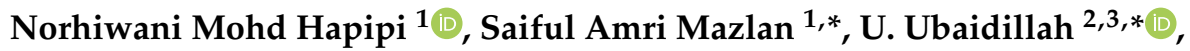 \\ Siti Aishah Abdul Aziz ${ }^{1}{ }^{\circledR}$, Muntaz Hana Ahmad Khairi ${ }^{1}{ }^{\circledR}$, Nur Azmah Nordin ${ }^{1}$ and \\ Nurhazimah Nazmi ${ }^{1}$ \\ 1 Engineering Materials and Structures (eMast) iKohza, Malaysia-Japan International Institute of Technology, \\ Universiti Teknologi Malaysia, Jalan Sultan Yahya Petra, Kuala Lumpur 54100, Malaysia; \\ hiwani87@gmail.com (N.M.H.); aishah118@gmail.com (S.A.A.A.); hana5700@gmail.com (M.H.A.K.); \\ nurazmah.nordin@utm.my (N.A.N.); nurhazimah@utm.my (N.N.) \\ 2 Mechanical Engineering Department, Faculty of Engineering, Universitas Sebelas Maret, Jl. Ir. Sutami 36A \\ Kentingan Jebres, Surakarta 57126, Indonesia \\ 3 National Center for Sustainable Transportation Technology (NCSTT), Bandung 40132, Indonesia \\ * Correspondence: amri.kl@utm.my (S.A.M.); ubaidillah_ft@staff.uns.ac.id (U.U.)
}

Received: 3 February 2020; Accepted: 27 February 2020; Published: 5 March 2020

\begin{abstract}
Chemically crosslinked hydrogel magnetorheological (MR) plastomer (MRP) embedded with carbonyl iron particles (CIPs) exhibits excellent magnetic performance (MR effect) in the presence of external stimuli especially magnetic field. However, oxidation and desiccation in hydrogel MRP due to a large amount of water content as a dispersing phase would limit its usage for long-term applications, especially in industrial engineering. In this study, different solvents such as dimethyl sulfoxide (DMSO) are also used to prepare polyvinyl alcohol (PVA) hydrogel MRP. Thus, to understand the dynamic viscoelastic properties of hydrogel MRP, three different samples with different solvents: water, DMSO, and their binary mixtures (DMSO/water) were prepared and systematically carried out using the oscillatory shear. The outcomes demonstrate that the PVA hydrogel MRP prepared from precursor gel with water shows the highest MR effect of 15,544\% among the PVA hydrogel MRPs. However, the samples exhibit less stability and tend to oxidise after a month. Meanwhile, the samples with binary mixtures (DMSO/water) show an acceptable MR effect of $11,024 \%$ with good stability and no CIPs oxidation. Otherwise, the sample with DMSO has the lowest MR effect of $7049 \%$ and less stable compared to the binary solvent samples. This confirms that the utilisation of DMSO as a new solvent affects the rheological properties and stability of the samples.
\end{abstract}

Keywords: magnetorheological plastomer; polyvinyl alcohol; dimethyl sulfoxide; corrosion; hydrogel

\section{Introduction}

Magnetorheological (MR) material is a material that consists of magnetic particles embedded in a carrier matrix. It has the capability of changing its properties by the application of external stimuli like a magnetic field due to the existence of magnetic particles. The MR material can be grouped into several types according to the difference of its carrier matrices, such as MR fluid (MRF), MR elastomer (MRE), MR foam, and MR gel (MRG). As the first developed MR material, MRF has been widely utilised in industrial applications such as vibration dissipation, damping, artificial muscles, drug delivery, valves, and actuators [1-4]. However, MRF exhibits several drawbacks, such as sedimentation and leaking problems that limit its application [5]. Thus, by replacing the fluid medium with a solid matrix, which is best known as MRE, the problems in MRF can be hindered [6]. Unlike the MRF, the magnetic particles inside MRE remain trapped in the matrix resulting in more stable mechanical properties of 
MRE. Unfortunately, MRE also has some drawback such as weak MR effects due to its hard nature of polymer matrix [7]. Therefore, a new class of MR material known as MR plastomer (MRP) has been developed recently due to its high MR effect and stability. As a new kind of intelligent MR material, MRP is prepared by dispersing micron-sized magnetic responsive particles into a low cross-linking polymer gel that has plastic characteristics such as hydrogel, polyurethane, paraffin, and polymer gel [8-11]. By referring to the high viscosity of polymer matrix, MRP becomes a promising candidate to substitute traditional MRF in some applications because no sedimentation of magnetic particles occurred [12]. Additionally, the magnetic particles inside the low cross-linked polymer matrix are moveable and not permanently trapped with the presence of an external magnetic field, which leads to a high MR effect in MRP.

At the early stage, the hydrogel matrix has been widely introduced as a carrying matrix to prepare MRP [7]. In general, hydrogel MRP comprises magnetic particles embedded inside a polymer matrix network swollen by a liquid solution like water and water-miscible organic solvent $[13,14]$. Hydrogel networks mainly include carrageenan, polysaccharides, agar, guar gum, and polymer derivatives such as polyvinyl alcohol (PVA). The MR behaviour of hydrogel MRP has been investigated extensively over the past decade and proven that the type of matrix strongly influences the mechanical properties of MRP. For instance, Mitsumata et al. [15,16], investigated the magnetic response of carrageenan magnetic hydrogel and found that the hydrogel exhibits negative MR effect. Moreover, the magnetic carrageenan gels display high reduction in storage modulus $\left(G^{\prime}\right)$ of $\sim 107$ Pa upon magnetisation. Meanwhile, Negami et al. [17] reported that the magnetic hydrogel using PVA as a matrix produced higher mechanical toughness compared to the carrageenan hydrogel. The yield point of magnetic PVA hydrogel was higher compared to that of the magnetic carrageenan with strain of 0.8 and 0.35 , respectively. The result shows that the magnetic PVA hydrogel possesses better mechanical strength compared to the magnetic carrageenan hydrogel. The giant MR behaviour and high yield point made the PVA hydrogel is suitable to be used as damping materials, sensors, and smart actuators.

PVA is a synthetic hydrophilic polymer, which is mechanically soft, biocompatible, high tensile modulus, high abrasion resistance, and high strength in compression that can be chemically and physically crosslinked [18-20]. Wu et al. [18] studied physically cross-linked PVA hydrogel MRP using the freezing-thawing method. The gelation of PVA hydrogel MRP arises through the formation of hydrogen bonding by crystallisation. They found that the PVA hydrogel MRP is a promising candidate for the manufacture of MR material as its tensile strength value was close to that of the natural rubber. The highest MR effect achieved by the sample was $230 \%$. Park et al. [20] compared the actuator behaviour of both chemically and physically cross-linked PVA hydrogel MRPs. The outcome demonstrated that the chemically cross-linked PVA hydrogel MRP exhibited lower stiffness and larger strains than those of the physically cross-linked PVA hydrogel MRP.

Although PVA hydrogel MRP has good MR behaviour and mechanical properties, several issues need to be considered, such as desiccation when exposed to air and degradation caused by the oxidation of magnetic particles. In other words, the oxidation occurs due to the high utilisation of water as an aqueous swelling solution in the preparation of hydrogel $[15,21-23]$. These disadvantages limit the application of PVA hydrogel MRP, especially for long-term applications in the engineering field. Thereby, the utilisation of water during the preparation of the material needs to be superseded so that it can be used in long-term activities. Thus, having the ability to potentially choose an ideal solvent to tailor the properties of the materials could be an extremely effective approach to improve the disadvantages stated previously. It is well known that the good mechanical properties and behaviour of PVA hydrogel MRP are influenced by the type and composition of solvents used [24].

Previously, several studies reported and proved that in conventional PVA hydrogel (without CIPs), organic solvent such as dimethyl sulfoxide (DMSO) is compatible as dispersing phase in the fabrication of PVA hydrogel solutions [25-28]. Shi and Han [25] discovered that DMSO is another great alternative to be used as the solvent in preparing PVA solutions compared to water. Cha et al. [27] proved that the type of solvent and mixing ratio affects the structure and mechanical properties of the 
material. They found that the PVA hydrogels prepared from mixed ratio of DMSO/water have better mechanical properties compared to those of prepared using other solvents. This finding also agreed well with the results by Gupta et al. [28], which prepared PVA hydrogels in the mixture of DMSO and water. In the study, the PVA hydrogels show interesting characteristics depending on the ratio of the DMSO and water, which lead to better mechanical properties compared to those prepared using other solvents.

Even though the utilisation of DMSO/water in the preparation of conventional PVA hydrogel have been widely investigated, to the best of our knowledge, many current research have yet to investigate the influence of different solvent in chemically cross-linked hydrogel MRP on its dynamic viscoelastic properties, particularly the storage modulus and damping properties. Considering the impact of solvent on the mechanical properties of conventional PVA hydrogel, it is expected that the type of solvents would greatly affect the dynamic properties of chemically cross-linked hydrogel MRP. Furthermore, by changing the solvents, the corrosion and desiccation problems are expected to diminish so that the application of PVA hydrogels MRP can be extended. Therefore, the main technical contribution of this paper is to experimentally investigate the influence of different solvents on the dynamic viscoelastic properties of chemically cross-linked hydrogel MRP. Three different solvents: water, DMSO, and their binary mixtures (DMSO/water) with different weight fractions of CIPs were used as experimental parameter and the dynamic properties of hydrogel MRP were obtained under oscillation mode test using a commercial rheometer. Hence, the influences of applied magnetic field and strain amplitude were investigated thoroughly. Additionally, the stability and corrosion of the as-prepared PVA hydrogel MRP were observed in a month, and the degradation of the samples are presented and discussed accordingly.

\section{Results and Discussions}

\subsection{Materials Characterisation}

\subsubsection{FTIR Measurement}

Figure 1 shows the FTIR spectra of the MRP samples with various solvents for samples S1-70, S2-70, and S3-70. The results are restricted to the MRP samples with CIPs concentration of $70 \mathrm{wt} . \%$.

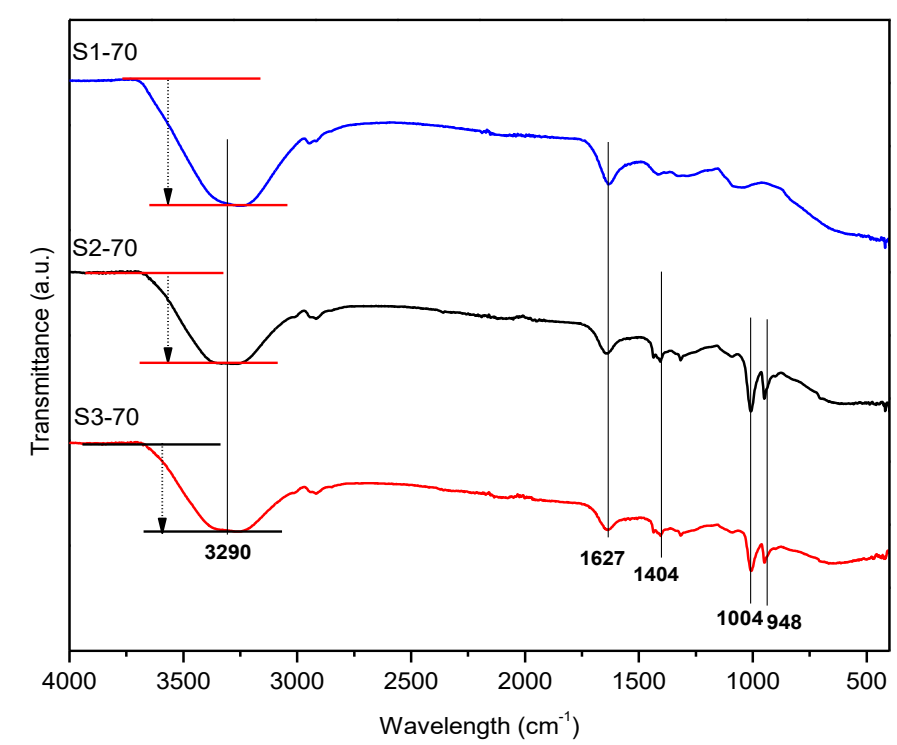

Figure 1. FTIR spectra of hydrogel magnetorheological plastomer (MRP) samples prepared with different types of solvents.

Figure 1 shows that five characteristics regions with strong transmittance peaks were observed at around 3300,1600, 1400, 1004, and $948 \mathrm{~cm}^{-1}$. The broad peak at around 3000 to $3500 \mathrm{~cm}^{-1}$ can 
be assigned to the hydrogen bond, $\mathrm{O}-\mathrm{H}$ stretching vibration region due to the intramolecular and intermolecular hydrogen bonds from the hydroxyl groups of PVA. The peak height at $3290 \mathrm{~cm}^{-1}$ for sample S1-70 increased compared to that of samples S2-70 and S3-70 due to the increase in O-H groups [26]. Moreover, the existence of peak at $3290 \mathrm{~cm}^{-1}$ belongs to the complexation of hydrogen bonding of $\mathrm{OH}$ groups from PVA borate ion as shown in Schemes 1 and 2. The result reveals that the PVA-borax having cross-linking network due to the complexation of PVA and borate ions. The peaks located at 1627 and $1404 \mathrm{~cm}^{-1}$ were associated with $\mathrm{C}=\mathrm{O}$ stretching and $\mathrm{C}-\mathrm{O}$ bending present in acetate groups, respectively. Meanwhile, the peaks at 1004 and $948 \mathrm{~cm}^{-1}$ can be assigned to the stretching vibration of $\mathrm{S}=\mathrm{O}$ for DMSO that only existed in Samples 2 and 3. As for Sample 3, the intensity of those two peaks ( $\mathrm{S}=\mathrm{O}$ stretching) was reduced due to $20 \mathrm{wt}$. \% of water.

\subsubsection{Vibrating Sample Magnetometer}

The magnetisation characterisation of the PVA hydrogel MRP with different solvents using a vibrating sample magnetometer (VSM) was successfully conducted as presented in Figure 2.

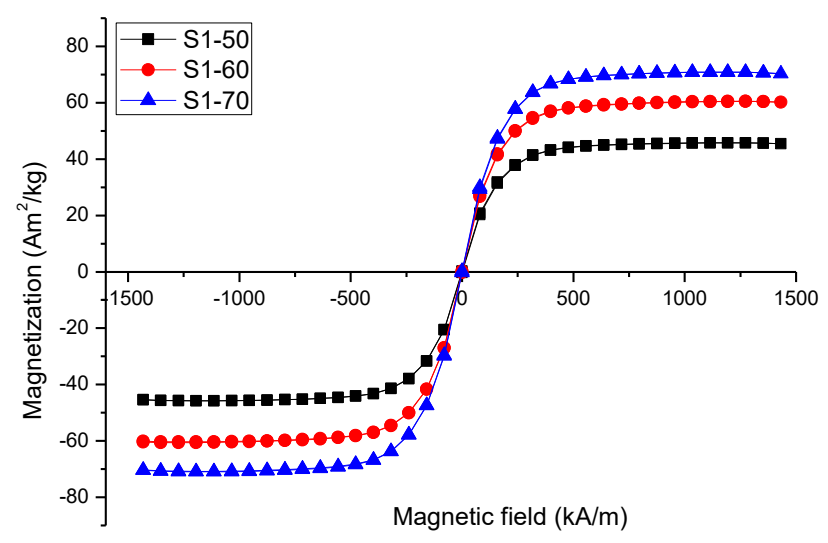

(a)

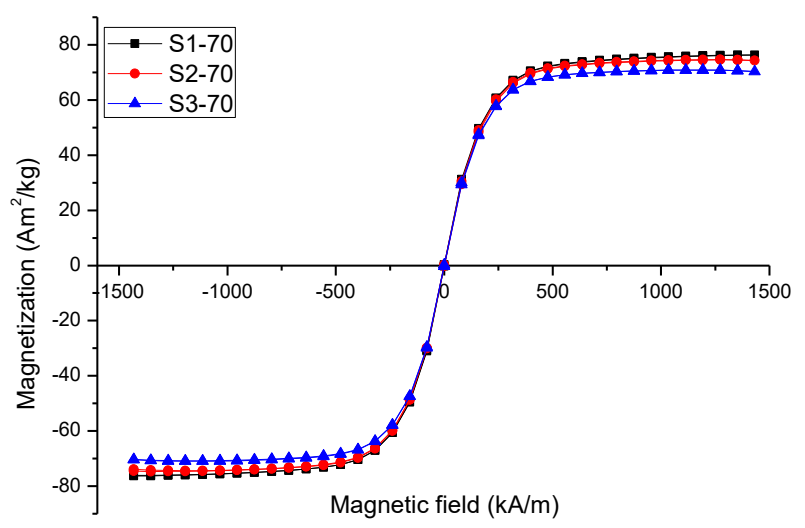

(b)

Figure 2. VSM profile of polyvinyl alcohol (PVA) hydrogel MRPs with (a) different carbonyl iron particles (CIPs) contents and (b) different types of solvent. 
Figure 2 shows that all the MRP samples exhibit hysteresis loops of typical soft magnetic material behaviour. Figure 2a displays the hysteresis loops of hydrogel MRP (PVA/DMSO) with different CIPs contents. The $M_{s}$ for hydrogel MRP sample (PVA/DMSO) with CIPs of 50, 60, and $70 \mathrm{wt} . \%$ was 44.45 , 60.69 , and $74.67 \mathrm{Am}^{2} / \mathrm{kg}$, respectively. The increase in $M_{s}$ with the increase in CIPs content is a common phenomenon in magnetic materials. The higher the $M_{s}$, the larger the responsive storage modulus $\left(G^{\prime}\right)$ of the samples towards the external stimuli, particularly magnetic field. Whilst, Figure $2 \mathrm{~b}$ presents that the hysteresis loops of PVA hydrogel MRP with different solvents and fixed CIPs amount of $70 \mathrm{wt} . \%$. The MRP samples display an unappreciable magnetic hysteresis as a prove that the type of solvent did not significantly change the magnetic saturation $\left(M_{s}\right)$. The $M_{s}$ for Samples S1-70, S2-70, and S3-70 was $76.21,74.67$, and $70.92 \mathrm{Am}^{2} / \mathrm{kg}$, respectively. The small difference in the $M_{s}$ value is due to the presence of different organic compounds in each of the samples that influence the reduction of the magnetic moment in CIPs inside each of the samples [2].

\subsection{Rheological Properties}

\subsubsection{Relative MR effect}

The MR effect is known as the magnetic field-dependent property and the key parameter to investigate the performance of MR material. MR effects are mainly originated from the interaction of magnetic particles or in this case, the CIPs, when the MRP samples are exposed to the magnetic field. The CIPs are magnetised and tend to form chain-like structures with the same magnetic field direction $[29,30]$. The field-induced magnetic forces between the particles restrict the deformation of polymer chains, resulting in the changes of $G^{\prime}$. Therefore, the reliance on the composition of CIPs and the type of solvents towards the dynamic mechanical properties of MRP was examined experimentally. Three MRP samples with various compositions of CIPs were prepared as shown in Table 1 . The influence of magnetic flux density on shear storage modulus is presented in Figure 3. All MRP samples were tested at a fixed frequency of $1 \mathrm{~Hz}$ and strain amplitude of $0.01 \%$. The applied external magnetic flux density was varied from 0 to $900 \mathrm{mT}$. To ensure the data consistency, all tests were run for three times, and the average values for both absolute and relative MR effect were calculated and presented in Table 1. The absolute MR effect is described as follow:

$$
\text { Absolute MR effect, } \Delta G^{\prime}=G^{\prime} \max -G_{0}^{\prime}
$$

While relative MR effect is the ratio of the absolute MR effect to the zero-field modulus, $G_{0}$ and usually expressed in percent's as below:

$$
\text { Relative MR effect }=\frac{G_{\max }-G_{0}}{G_{0}} \times 100 \%
$$

where $G_{0}$ is the zero-field modulus, and $G_{\max }$ is the maximum modulus achieved in the presence of magnetic field. 


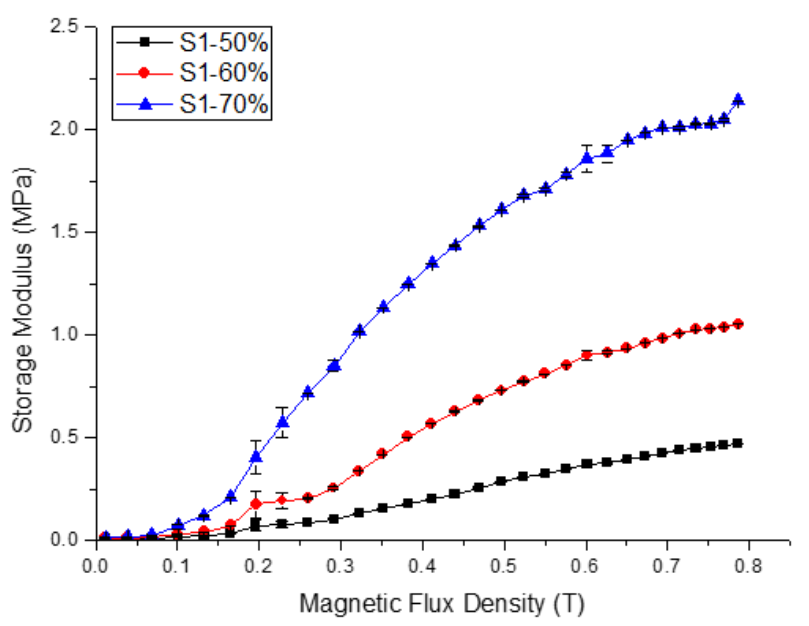

(a)

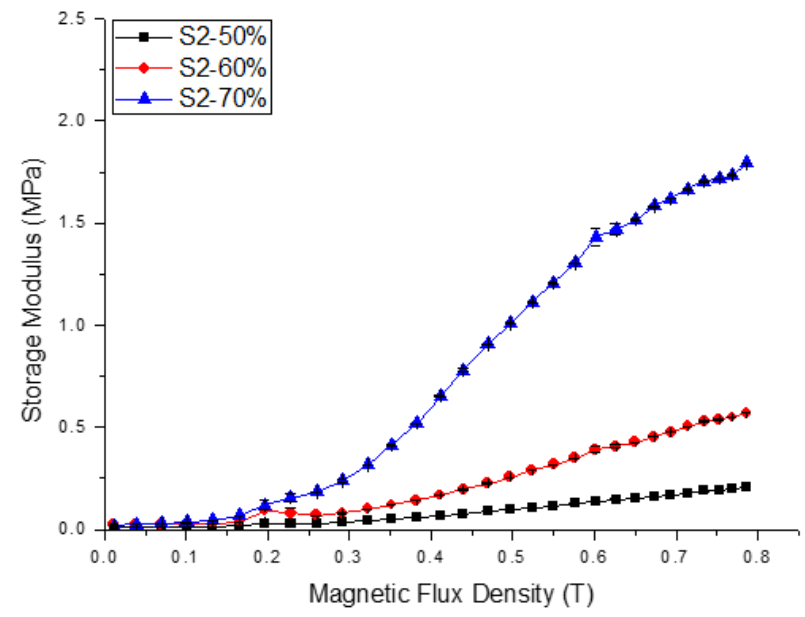

(b)

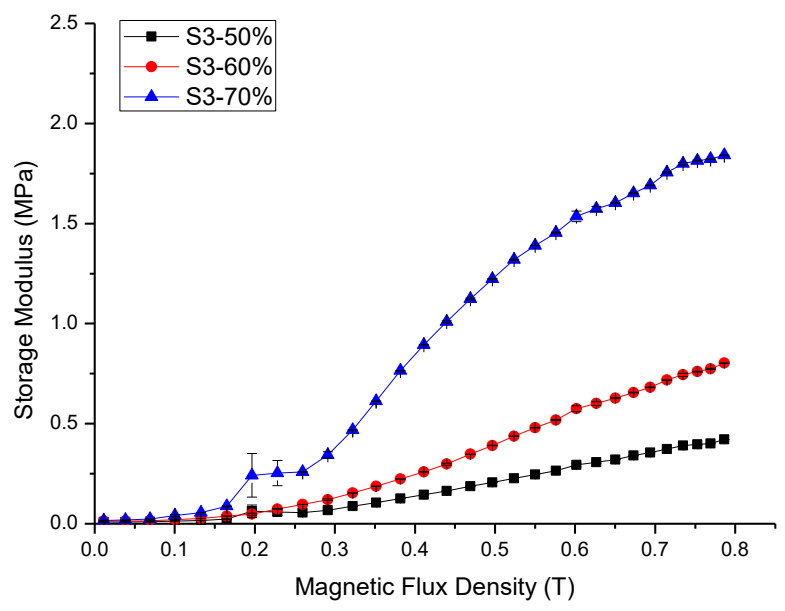

(c)

Figure 3. Shear storage modulus as a function of magnetic flux density with different CIPs compositions for (a) Sample 1, (b) Sample 2 and (c) Sample 3. 
Table 1. The absolute and relative magnetorheological (MR) effect hydrogel MRP with different solvents and CIPs content.

\begin{tabular}{|c|c|c|c|c|c|c|c|c|c|c|c|c|c|}
\hline \multirow{2}{*}{ Sample } & \multicolumn{5}{|c|}{ Initial Storage Modulus, $\mathbf{G}_{0}^{\prime}$} & \multicolumn{5}{|c|}{ Max. Storage Modulus, $G_{\text {max }}^{\prime}$} & \multirow{2}{*}{$\begin{array}{c}\text { Avg. Absolute MR } \\
\text { Effect }\end{array}$} & \multirow{2}{*}{$\begin{array}{c}\begin{array}{c}\text { Avg. Relative MR } \\
\text { Effect }\end{array} \\
\%\end{array}$} & \multirow[b]{2}{*}{$\mathbf{b}_{p}$} \\
\hline & 1 & 2 & 3 & Mean & a $\mathrm{SD}$ & 1 & 2 & 3 & Mean & ${ }^{a}$ SD & & & \\
\hline $\mathrm{S} 1-50$ & 0.008 & 0.008 & 0.009 & 0.008 & 0 & 0.472 & 0.473 & 0.473 & 0.473 & 0 & 56.307 & 5631 & \multirow{3}{*}{0.002} \\
\hline S2-50 & 0.012 & 0.006 & 0.012 & 0.010 & 0.001 & 0.206 & 0.207 & 0.207 & 0.207 & 0 & 19.939 & 1994 & \\
\hline S3-50 & 0.008 & 0.008 & 0.008 & 0.008 & 0.001 & 0.421 & 0.422 & 0.421 & 0.421 & 0 & 50.231 & 5023 & \\
\hline S1-60 & 0.009 & 0.008 & 0.010 & 0.009 & 0.004 & 1.055 & 1.055 & 1.055 & 1.055 & 0 & 115.401 & 11,540 & \multirow{3}{*}{$\begin{array}{c}< \\
0.001\end{array}$} \\
\hline S2-60 & 0.025 & 0.021 & 0.024 & 0.023 & 0.002 & 0.570 & 0.572 & 0.570 & 0.571 & 0.001 & 23.442 & 2344 & \\
\hline S3-60 & 0.013 & 0.011 & 0.013 & 0.012 & 0.002 & 0.802 & 0.803 & 0.802 & 0.802 & 0 & 63.426 & 6343 & \\
\hline $\mathrm{S} 1-70$ & 0.013 & 0.015 & 0.013 & 0.014 & 0 & 2.140 & 2.140 & 2.140 & 2.140 & 0 & 155.448 & 15,544 & \multirow{3}{*}{$\begin{array}{c}< \\
0.001\end{array}$} \\
\hline $\mathrm{S} 2-70$ & 0.026 & 0.023 & 0.026 & 0.025 & 0.001 & 1.791 & 1.792 & 1.791 & 1.792 & 0.001 & 70.491 & 7049 & \\
\hline S3-70 & 0.017 & 0.016 & 0.017 & 0.017 & 0.001 & 1.841 & 1.841 & 1.841 & 1.841 & 0 & 110.242 & 11,024 & \\
\hline
\end{tabular}


Figure 3 shows that the hydrogel MRP samples exhibit MR effect behaviour, where the $G^{\prime}$ increased consistently with the increase in magnetic flux density. A steep increase in $G^{\prime}$ appears for all samples when the magnetic flux density increased from 0 to $900 \mathrm{mT}$. However, Figure 3a shows that the increase in $G^{\prime}$ for Sample 1 was more obvious compared to that of the Samples 2 and 3 in Figure 3b,c. For examples, the $G^{\prime}$ of samples with CIPs concentration of $70 \mathrm{wt} . \%$ (S1-70), displays the highest increase of 2.14 MPa at $900 \mathrm{mT}$. Meanwhile for Samples S2-70 and S3-70, the $G^{\prime}$ increased up to 1.79 and $1.84 \mathrm{MPa}$, respectively. Besides, the saturated $G^{\prime}$ is highly dependent on the composition of CIPs and significantly improved the MR effect. From Figure 3, the increment of $G^{\prime}$ for all samples with a higher concentration of CIPs was more obvious compared to that of the samples with smaller concentrations. From these results, the average absolute and relative MR effect for all samples were calculated and summarised in Table 1. The relative MR effect of each sample can be calculated using.

From Table 1, the PVA hydrogel MRP with profoundly filled CIPs accomplished the highest relative MR effect. For example, the MR effect of S1-50, S1-60, and S1-70 was 5631, 11,540, and 15,544\%, respectively, of which the MR effect increased with increasing of CIPs percentage. On the other hand, the maximum relative MR effect of samples with the highest concentrations of CIPs: S1-70, S2-70, and S3-70 increased to $15,544,7049$, and $11,024 \%$, respectively. These findings agree with the results by Wang et al. [31], which found that the concentration of magnetic particles effectively influence the MR effect of the MR materials.

Table 1 also shows that different solvents affect the MR effect of the hydrogel MRP. The MR effect for Sample 2 was always the lowest compared to that of Samples 1 and 3 even though the concentration of CIPs was increased. In contrast, Sample 1 especially Sample S1-70 shows the highest MR effect among the samples due to the highest hydroxyl group caused by the highest amount of water content that soften the matrix. Thus, the CIPs were easily moved in Sample S1-70 to form the chain-like structures that restrict the polymer chains of PVA when subjected to the magnetic field. Sample S2-70 has a lower MR effect compared to Sample S3-70 because the utilisation of DMSO as the whole percentage of solvent contributed to the stiffness of the matrix. Additionally, based on Table 1 , one can see that CIPs percentages and solvents type, with $p$-values less than 0.05 , significantly affect the performance of PVA hydrogel MRPs toward MR effect. According to Krumova et al. [32], the PVA hydroxyl groups mainly consist of the hydrogen bonds that provide a major contribution towards the stiffness of the polymer chains. By introducing the DMSO as the solvent, the hydrogen bonding interaction diminished due to the existence of covalent bonding from sulphur and oxygen bonding resulting in the increment of the matrix stiffness. However, the stiffness of the matrix can be reduced by introducing the binary mixtures of DMSO/water as exhibited in Sample S3-70.

\subsubsection{Strain Amplitude Sweep}

To understand the influence of different solvents on the viscoelastic properties of PVA-based hydrogel MRPs, strain amplitude tests under oscillatory shear rheometry were performed to determine the linear viscoelastic range of the MRPs. The LVE range is very important for viscoelastic materials like MRP to further comprehend the relationship between the microstructure rupture of MRP induced by external stimuli such as strain amplitude. Figure 4 shows the strain amplitude dependence of MRP with different solvent types on the $G^{\prime}$ under 0 and magnetic field of $600 \mathrm{mT}$. The $G^{\prime}$ is an important parameter to characterise the ability of the MRP to store energy after shear deformation. 


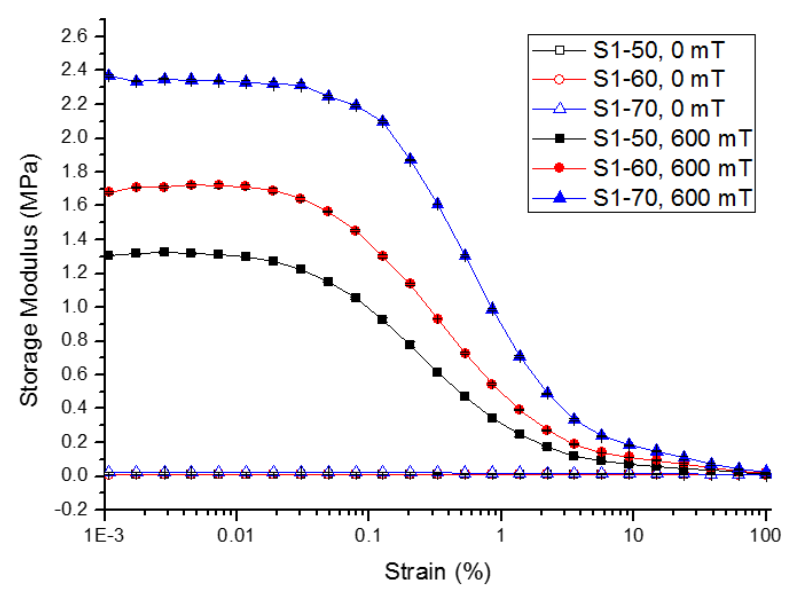

(a)

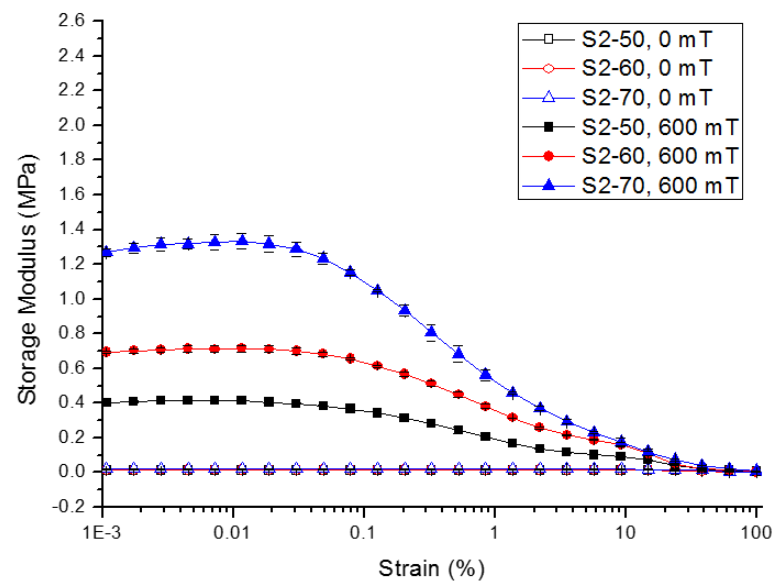

(b)

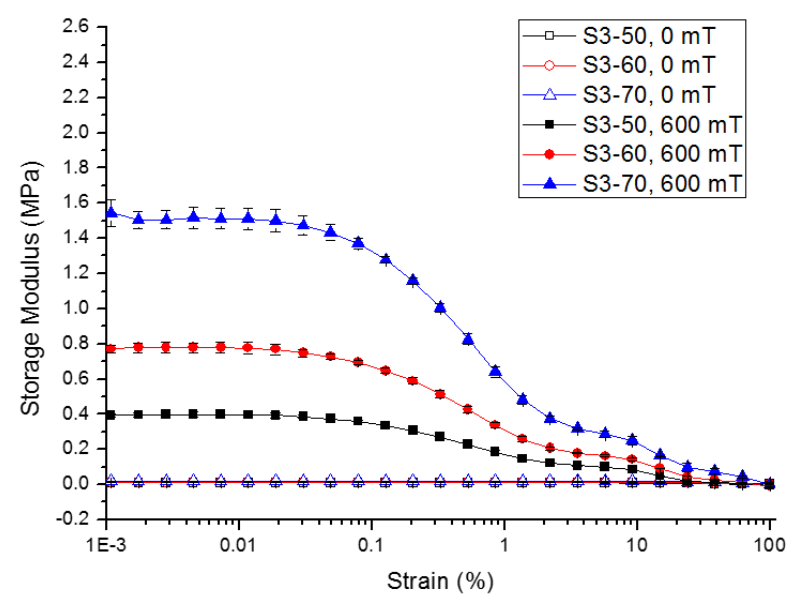

(c)

Figure 4. The dependencies of $G^{\prime}$ (a) Sample 1 (b) Sample 2, and (c) Sample 3 as a function of strain amplitude. ( $*$ at constant frequency, $\mathrm{f}=1 \mathrm{~Hz}$ ).

Figure 4 shows that at the off-state condition $(0 \mathrm{mT})$, the $G^{\prime}$ remained unchanged with the increment of strain amplitude. All MRP samples approximately behave as linear viscoelastic materials as the $G^{\prime}$ is independent of the strain amplitude at the off-state condition. Interestingly, at this condition, the $G^{\prime}$ of MRP samples (see Figure $4 \mathrm{a}-\mathrm{c}$ ), was not increased even when many CIPs were embedded in 
the matrix. In other words, CIPs in the MRP without magnetic field were randomly distributed in the polymer matrix. A similar trend was also observed in the study reported by Mitsumata et al. [22]. At the on-state condition $(600 \mathrm{mT})$, the $G^{\prime}$ increased remarkably with the CIPs content for all MRP samples as shown in Figure $4 a-c$. The $G_{\max }$ achieved by Samples S1-70, S2-70, and S3-70 was 2.370, 1.239 , and $1.576 \mathrm{MPa}$, respectively. The increment of $G^{\prime}$ indicates that in the presence of a magnetic field, the CIPs strengthen the matrix by reducing the distance between the particles. The strong dipole-dipole interaction between the CIPs is due to the magnetisation of CIPs to form chain-like structures attracted to each other inside the polymer chains.

Table 2 depicts the zero-field modulus $\left(\mathrm{G}^{\prime}{ }_{0}\right)$, maximum modulus $\mathrm{G}^{\prime}{ }_{\max }$, and magneto-induced modulus $\Delta \mathrm{G}^{\prime}$ of all samples with $70 \mathrm{wt} . \%$ of CIPs. Sample S1-70 has the highest $\Delta \mathrm{G}^{\prime}$ of $2.35 \mathrm{MPa}$, followed by Samples S3-70 and S2-70. It could be explained by the formation of hydroxyl group inside the matrix. The strong evidence of this statement is supported by the FTIR results in Figure 1. Due to the highest amount of hydroxyl group, the CIPs inside the matrix are easy to move, magnetise, and form chain-like structures when subjected to the magnetic field, therefore dramatically increased the $\Delta \mathrm{G}^{\prime}$. Meanwhile, Sample S2-70 has the lowest $\Delta \mathrm{G}^{\prime}$ of $1.21 \mathrm{MPa}$ because it was made up of DMSO that increased the rigidity of the sample and reduced the elasticity of the MRP. Then, in Sample S3-70 (the binary mixtures of DMSO/water in a ratio of 80:20), the rigidity of the sample was reduced and the $\Delta \mathrm{G}^{\prime}$ was improved.

Table 2. The zero-field modulus $\left(\mathrm{G}_{0}^{\prime}\right)$ maximum modulus $\left(\mathrm{G}_{\max }\right)$ and magneto-induced modulus $\Delta \mathrm{G}^{\prime}$.

\begin{tabular}{cccccccccccc}
\hline \multirow{2}{*}{ Sample } & \multicolumn{4}{c}{ Initial Storage Modulus, $\mathrm{G}^{\prime}{ }_{\mathbf{0}}$} & \multicolumn{4}{c}{ Max. Storage Modulus, $\mathbf{G}_{\text {max }}$} & \multicolumn{3}{c}{$\boldsymbol{\Delta G}^{\prime}$} \\
\cline { 2 - 12 } & $\mathbf{1}$ & $\mathbf{2}$ & $\mathbf{3}$ & $\mathbf{M e a n}$ & $\mathbf{S D}$ & $\mathbf{1}$ & $\mathbf{2}$ & $\mathbf{3}$ & Mean & SD & MPa \\
\hline S1-70 & 0.021 & 0.019 & 0.02 & 0.020 & 0.001 & 2.366 & 2.357 & 2.387 & 2.370 & 0.015 & 2.35 \\
S2-70 & 0.032 & 0.036 & 0.034 & 0.034 & 0.002 & 1.236 & 1.236 & 1.246 & 1.239 & 0.006 & 1.21 \\
S3-70 & 0.02 & 0.019 & 0.018 & 0.019 & 0.001 & 1.665 & 1.562 & 1.501 & 1.576 & 0.083 & 1.56 \\
\hline
\end{tabular}

Additionally, at the on-state condition, the $G^{\prime}$ of the MRP samples decreased sharply with the increasing of strain amplitude. The sharp decrement of $G^{\prime}$ was more pronounced for the MRP sample with $70 \mathrm{wt} . \%$ of CIPs when the strain exceeded $0.03 \%$. This phenomenon is common in MR materials and called as a Payne effect. Generally, the Payne effect is the destruction or breakage of the magnetic particle chains due to the large amplitude strain and depends much on the CIPs content in the matrix [33]. Below critical strain $\left(\gamma_{c}=0.03 \%\right)$, the material is still in within the LVE range where the materials are stable, and the chain structures have not been destroyed. Correspondingly, the range above $\gamma_{c}$ is known as non-linear LVE range, where the disruption of chains starts to occur. Figure 4 shows that the sort of solvents has almost no significant effect on the LVE range of MRP, however, the LVE can be enormously changed by the content of CIPs. Thus, from the results, the LVE range for MRP samples with various kind of solvents can be regarded as $0-0.03 \%$.

\subsubsection{Damping Properties}

The matrix and composition of CIPs also play a crucial role in the determination of damping properties of MRP. Damping properties reflect the capability of the material to dissipate energy in a vibrating system known as loss factor. The shear strain dependency of the loss factor is displayed in Figure 5. 


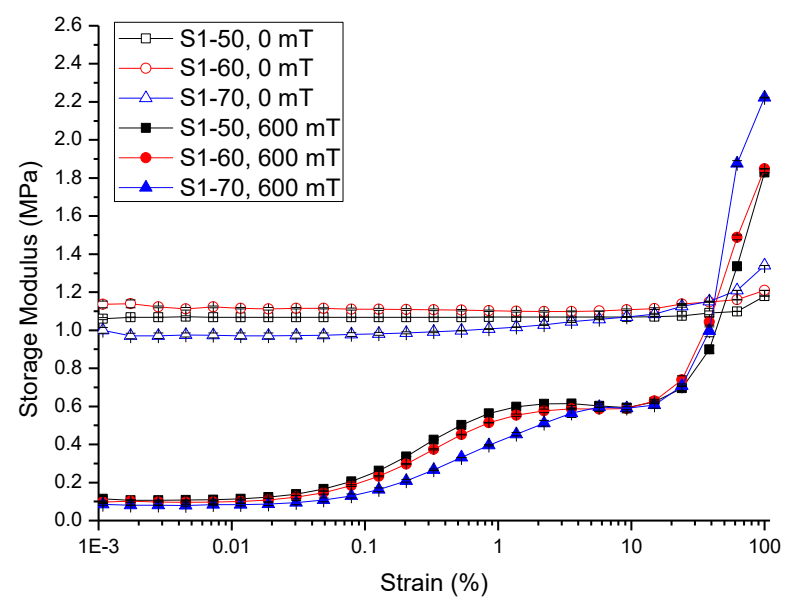

(a)

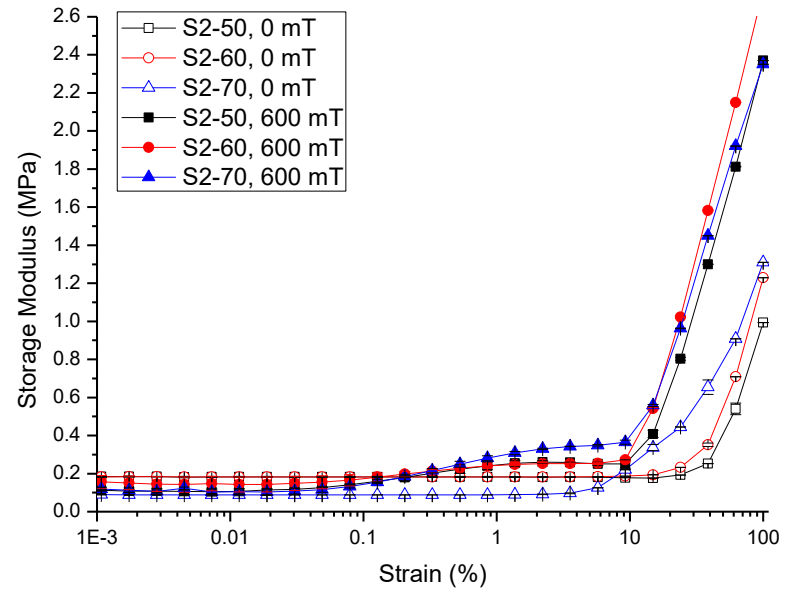

(b)

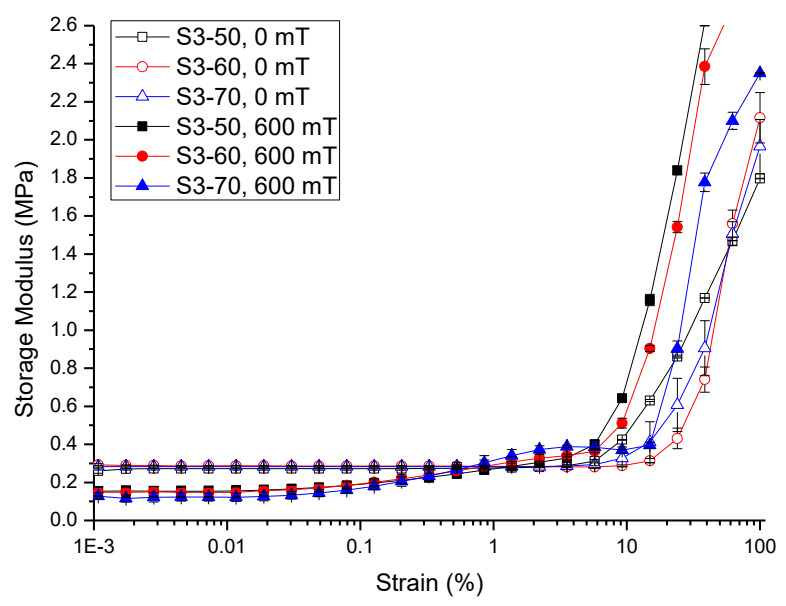

(c)

Figure 5. The dependencies of loss factor (a) Sample 1, (b) Sample 2 and (c) Sample 3 as a function of strain amplitude. ( ${ }^{* a t}$ constant frequency, $\mathrm{f}=1 \mathrm{~Hz}$ ).

From Figure 5, the loss factor for all MRP samples increased with increasing strain amplitude up to $100 \%$. The off-state loss factor was higher than the on-state loss factor and shows linear variation with the strain amplitude. Generally, the dissipation of energy is caused by the interfacial slipping 
within the magnetic particles and the movement of polymer chains in the polymer [34]. However, in the absence of magnetic fields, the damping properties of materials is hinged on the polymer chains of the matrix. As noticed, Sample 1 shows the highest off-state loss factor at $\sim 1.2 \%$ when the CIPs content was $50 \mathrm{wt} . \%$. The astounding loss factor of Sample 1 compared to Samples 2 and 3 may be due to the liquid-like nature of the sample. The liquid-like nature of Sample 1 may be attributed to the higher formation of hydrogen bonds between the -OH groups of PVA, which will cause the polymer chains (soft segments) move more easily without being blocked in the matrix. Therefore, the loss factor was larger for Sample 1 compared to that of Samples 2 and 3 dues to the most minimal formation of hydrogen bonds. Meanwhile, Samples 2 and 3 can be considered as solid-like MRP because they comprise more hard segments compared to soft segments. The off-state loss factor of solid-like MRPs is lower because the interfacial slipping between the polymer chains barely happens due to stronger interactions between the polymer chains [35].

Upon the application of magnetic field, the behaviour of the on-state loss factor was obviously different from the off-state loss factor. Instead of long plateau up to the higher strain of $\sim 10 \%$, the on-state loss factor shows interesting behaviour. As illustrated in Figure $5 \mathrm{a}-\mathrm{c}$, the on-state loss factor was always lower than the off-state factor for all MRP samples. The on-state loss factor of MRP samples started to level off at very small strain up to $0.1 \%$ and increased with increasing strain amplitude. Interestingly, at the strain of greater than $0.1 \%$, the plateaued region started to increase again. The increased loss factor can be related to the strain hardening of polymer chains as the strain increased. Strain hardening is a strengthening behaviour of the material during a large strain deformation caused by the large-scale orientation of polymer chains molecules. The increased loss factor at the higher strain caused by strain hardening is due to the rupture of polymer chains that increased the dissipation energy. This phenomenon was observed in plastic materials as reported in previous research [36]. Sample 1 shows larger strain hardening compared to Samples 2 and 3 because the liquid-like nature of the matrix caused more dislocation of the polymer chains, thus increased the loss factor.

Furthermore, Figure 5 shows that the loss factor decreased in the presence of the magnetic field; therefore, it is proven that less heat dissipation occurred during deformation due to stronger particle interactions. This is because, in the presence of magnetic field, the CIPs are magnetised and the magnetic interaction between the particles is increased. Thus, the interfacial slipping within the particles are reduced. Basically, the on-state loss factor is depending on the interaction between the magnetic particles. Therefore, with the presence of magnetic field, the magnetic force between the CIPs increases, thereby reducing the distance between CIPs which results in the decrement of interfacial slipping. Moreover, the loss factor of MRP samples also decreased with increasing CIPs concentration either in the off-state or the on-state conditions.

\subsection{Desiccation and Corrosion Observation}

Material stability is also an important criterion for assessing the performance of MR materials. As previously mentioned, hydrogel MRPs have a higher possibility for the particles to sediment or corrode over time. To prove the desiccation event occurred in the samples, three Samples-S1-70, S2-70, and S3-70-were cut into spherical disk with $20 \mathrm{~mm}$ in diameter and left exposed to the air for $24 \mathrm{~h}$. The photograph of all samples is displayed in Figure 6. 


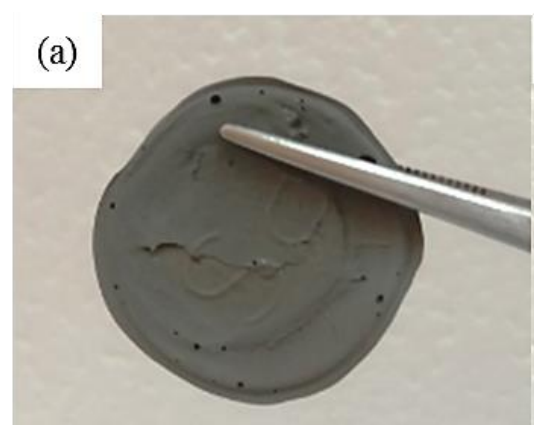

After $24 \mathrm{hrs}$ (b)

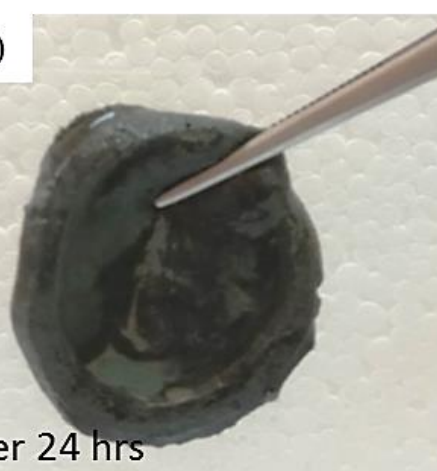

(c)

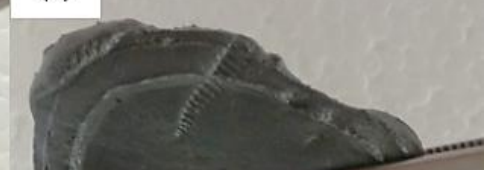

After $24 \mathrm{hrs}$

Figure 6. The desiccation observations after $24 \mathrm{~h}$ for sample: (a) S1-70, (b) S2-70, and (c) S3-70.

Figure 6a shows that Sample S1-70 was desiccated and lost its softness (solidified) after exposed to open air for $24 \mathrm{~h}$. The desiccation occurred because water starts to evaporate as the polymer structures are chemically less stable [37]. However, in Samples S2-70 and S3-70, the softness remained after $24 \mathrm{~h}$ of exposure to air as shown in Figure $6 \mathrm{~b}, \mathrm{c}$, respectively. It can be concluded that, with the addition of DMSO as a solvent during the dispersing phase, the solidification problems can be reduced. Other serious problems that mentioned previously, is the degradation of the samples due to the oxidation of CIPs that lead to corrosion and cause limitation in practical use. The photograph in Table 3 shows the degradation of the hydrogel MRP samples after a month due to the oxidation of CIPs that lead to corrosion problem.

Table 3 shows that Sample 1 (S1-50, S1-60, and S1-70) formed a yellowish layer due to the oxidation of CIPs. Furthermore, the yellowish layer was higher for the sample with the lowest content of CIPs (S1-50). Samples S1-50, S1-60, and S1-70 tended to corrode due to the existence of water molecules that contribute to the oxidation process of the CIPs. For Sample 2 (S2-50, S2-60, and S2-70), no yellowish layer was formed after a month, however, the CIPs settling was obvious for Sample S2-50. The oxidation of CIPs did not occur in Sample 2 as the samples were prepared with the DMSO without the existence of water molecules. Sample 3 (S3-50, S3-60, and S3-70) remained unchanged after a month. In fact, particles settling did not occur in Sample 3, which indicates that the stability of MRP is superior in the hydrogel MRP prepared from the binary mixtures of DMSO/water. The corrosion occurred in Sample 1 due to the higher water molecule compared to Samples 2 and 3. The numbers of water molecules inside the PVA solutions followed the order of, PVA hydrogel (water) $>$ PVA hydrogel (DMSO/water) > PVA hydrogel (DMSO). This result shows a good agreement with the results obtained using molecular dynamic simulations studied by Shi et al. [25], which declared that water is the poorest solvent compared to DMSO for PVA hydrogel. 
Table 3. Photograph images of MRP samples conditions after a month: Sample 1, Sample 2, and Sample 3 with different concentrations of CIPs.

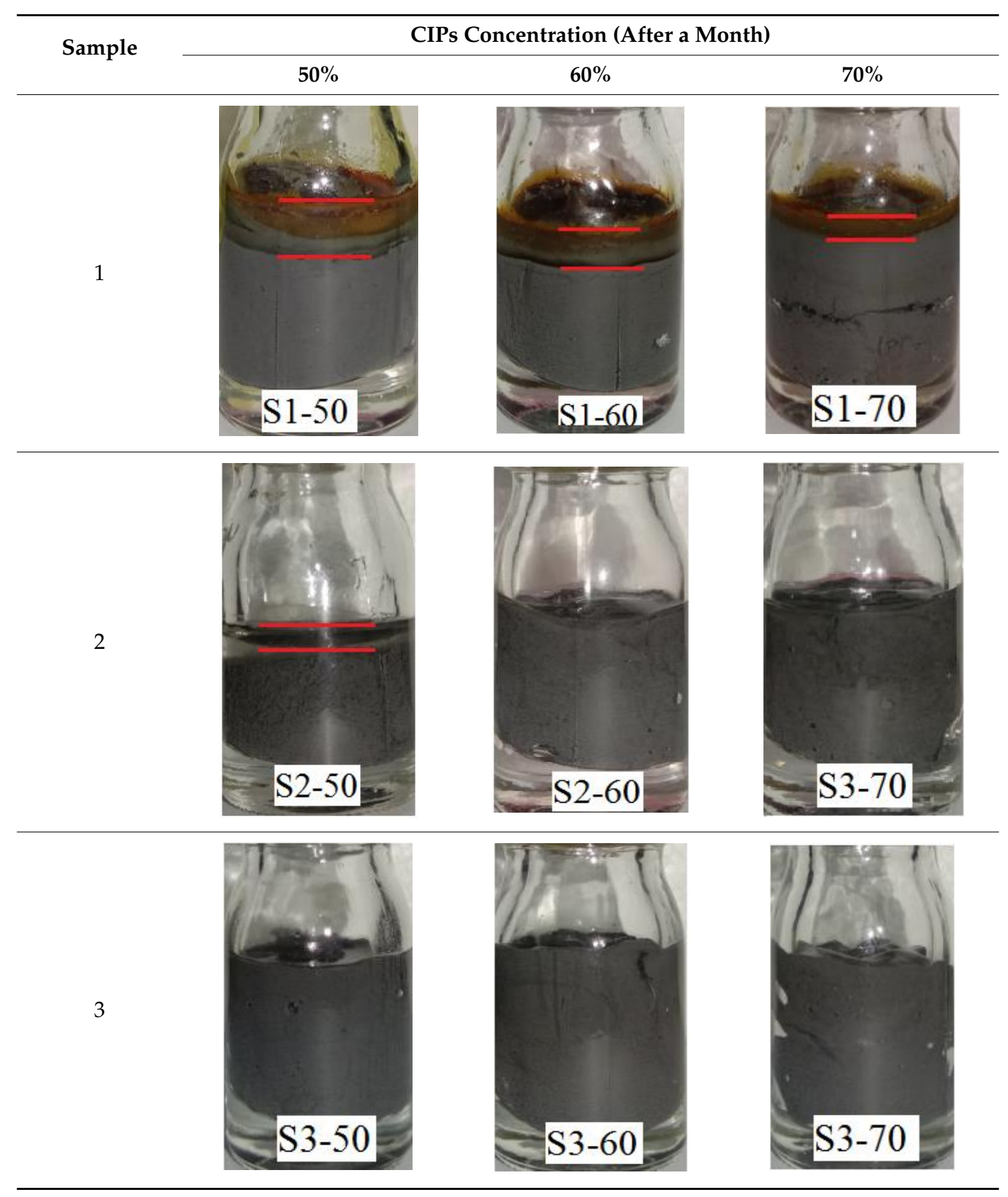

\section{Materials and Methods}

\subsection{Material}

In this study, the high molecular weight PVAs ( $\geq 98.0 \%$ hydrolysed, approximate molecular weight of 60,000) were purchased from Merck Company, Germany. Sodium tetraborate decahydrate (borax), 20 Mule Team Borax ${ }^{\mathrm{TM}}$ was purchased from a drug store and used as a cross-linking agent. As a solvent, DMSO brand ChemAR was supplied by Systerm Chemicals. CIPs with a size of $\sim 5 \mu \mathrm{m}$ were procured by BASF (CC model). Deionised water was used to prepare the aqueous solution. 


\subsection{Preparation of PVA MRP}

Firstly, a PVA solution of $7.5 \%(w / v)$ was prepared by dissolving $8.1 \mathrm{~g}$ of PVA beads in $100 \mathrm{~mL}$ of deionised water at $80^{\circ} \mathrm{C}$ using a hotplate for $2 \mathrm{hr}$. Similar steps were repeated with a different mixture of organic solvent as illustrated in Table 4 . The mixture was then cooled down to room temperature after completely dissolved. Secondly, the borax solution of $3 \%(w / v)$ was prepared by mixing the powder with deionised water and act as the cross-linking agent. CIPs with different concentrations of 50,60 , and $70 \mathrm{wt} . \%$ were added into each of PVA solutions. Next, the PVA solutions that contain CIPs were chemically cross-linked using borax solution with constant stirring using mechanical stirrer for $10 \mathrm{~min}$. The mixture was kept overnight to obtain a uniform MRP sample.

Table 4. The compositions of hydrogel MRP with different types of solvents.

\begin{tabular}{cccc}
\hline & Sample & CIPs Content, wt.\% & PVA Content, wt.\% \\
\hline \multirow{2}{*}{ Sample 1 (PVA/water) } & S1-50 & 50 & 50 \\
\cline { 2 - 4 } & S1-60 & 60 & 40 \\
\cline { 2 - 4 } & S1-70 & 70 & 30 \\
\hline \multirow{2}{*}{ Sample 2 (PVA/DMSO) } & S2-50 & 50 & 50 \\
\cline { 2 - 4 } & S2-60 & 60 & 40 \\
\cline { 2 - 4 } Sample 3 & S2-70 & 70 & 30 \\
\cline { 2 - 4 } (PVA/DMSO:water) & S3-50 & 50 & 50 \\
\cline { 2 - 4 } & S3-60 & 60 & 30 \\
\hline
\end{tabular}

\subsection{Mechanism of Chemically Crosslinking}

Unlike the physically cross-linked hydrogel MRPs, chemically cross-linked hydrogel MRPs arises from the presence of reversible chemical crosslinks of polyol-containing polymers that respond to the borate ion [38]. The chemistry applicable to the polyol-borate systems is shown in Figure 7. This interaction shows a di-diol complexation.

Scheme 1:

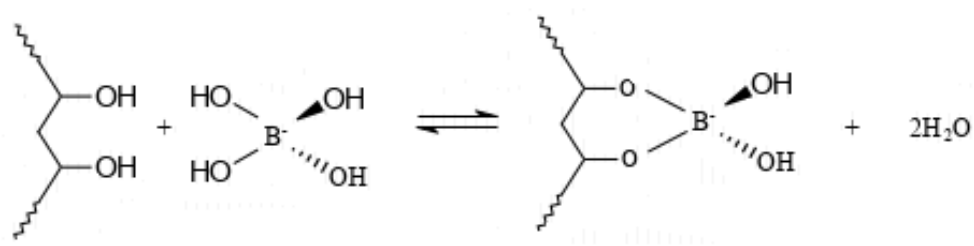

Scheme 2:
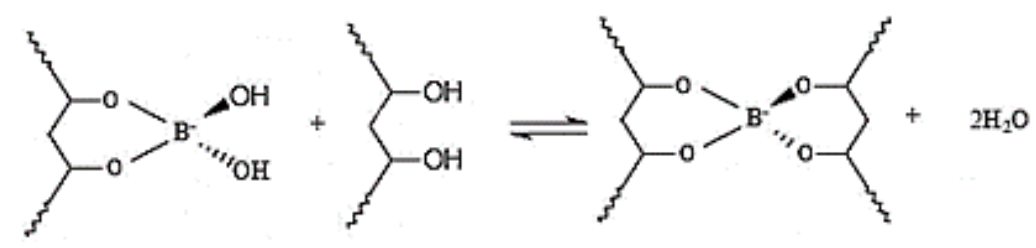

Figure 7. The cross-linking structure of compound formation between PVA hydrogel and borax.

From Scheme 1 in Figure 7, the borate ions react with the diol unit of polymer to form mono diol. In Scheme 2, the monodiol reacts with a second diol unit of the polymer to form di-diol. A hydrogen bond is connected between the boron sites and PVA chains. The hydrogen bond is reversible and much lower than the chemical bond that leads to the self-healing ability of the materials after cleaving or shearing by force [38]. 


\subsection{Characterization and Rheological Testing}

The magnetic characteristics of MRP samples was evaluated using a VSM (Microsense 7404) at room temperature. The test was carried out using a broad range of magnetic field up to $1500 \mathrm{k} / \mathrm{Am}$. The molecular structure of the PVA solutions was measured using FTIR (Bruker FTIR) spectrometer in the range of $4000-500 \mathrm{~cm}^{-1}$ at ambient temperature. Meanwhile, the dynamic mechanical properties of the hydrogel MRPs sample were measured using a rheometer (Model: MCR 302 Anton Paar) at controlled temperature of $25^{\circ} \mathrm{C}$. In this study, an oscillation mode was used to study the dynamic properties, strain amplitude, and magneto-field sweep test. The rheological testing for each sample has been repeated three times in order to reassure the consistency of the values. The mean, standard deviation, percentage of error, and the one-way ANOVA have been quantitatively calculated and presented. In one-way ANOVA analysis, the value of $p=0.05$ was used to determine the variable (i.e., types of solvent) were statistically significant. Moreover, the stability test of MRP samples was thoroughly investigated and discussed in detail.

\section{Conclusions}

The present study was designed to determine the opportunities of using different solvents in the fabrication of PVA hydrogel MRP as an alternative to solve corrosion and desiccation problems as mentioned in previous studies. Three kinds of hydrogel PVA MRPs were prepared that contained water, DMSO, and their binary mixtures (DMSO/water) with ration of 80:20 by weight were prepared. Different percentages of CIPs $(50,60$, and $70 \mathrm{wt} . \%)$ were added to each sample, making nine samples in total. The field-dependent rheological characteristics of all samples were experimentally investigated, and the corrosion event was observed for a month. The results show that the PVA hydrogel MRP prepared from water has the highest MR effect of 15,544\% (Sample S1-70) with a CIPs concentration of 70 wt.\%. Although Sample 1 has a higher MR effect than Samples 2 and 3, the presence of high-water molecules contributed to corrosion and desiccation problems. In contrast, Sample 2 (S2-70) and 3 (S3-70) have lower MR effects of 7049\% and 11,024\%, respectively, compared to Sample 1. However, Samples 2 and 3 have a better stability and no corrosion was observed after one month. In addition, the results show that the rheological properties of hydrogel PVA MRP samples are strongly influenced by the types of solvents and the CIPs concentration.

Author Contributions: Conceptualization, N.M.H., S.A.M., S.A.A.A., U.U.; Methodology, N.M.H., M.H.A.K, U.U.; Validation, N.M.H., S.A.M., S.A.A.A., N.N., N.A.N.; Writing-Original Draft Preparation, N.M.H.; Writing-Review \&Editing, N.M.H., S.A.M., S.A.A.A., U.U., N.N., M.H.A.K., N.A.N. All authors have read and agreed to the published version of the manuscript.

Funding: This work is supported and financial funded by Professional Development Research University (PDRU) grant (Vot No. 04E02). This work also supported by Universiti Teknologi Malaysia under Transdisciplinary Research (UTM-TDR) Grant, Vot No: 07G13. Authors also acknowledge Universitas Sebelas Maret for the financial aid through Hibah Program Hilirisasi Hasil Riset 2020 as well as National Center for Sustainable Transportation Technology (NCSTT) for the partial funding through IIE00000078-ITB-1.

Conflicts of Interest: The authors declare that there is no conflict of interest.

\section{References}

1. Garg, D.P.; Anderson, G.L. Research in active composite materials and structures: An overview. Smart Struct. Mater. Act. Mater. Behav. Mech. 2000, 3992, 2-12.

2. Ahmadian, M.; Poynor, J.C. An evaluation of magneto rheological dampers for controlling gun recoil dynamics. Shock Vib. 2001, 8, 147. [CrossRef]

3. Ladipo, I.L.; Fadly, J.D.; Faris, W.F. Characterization of Magnetorheological Elastomer (MRE) Engine Mounts. Mater. Today Proc. 2016, 3, 411-418. [CrossRef]

4. Bazinenkov, A.M.; Mikhailov, V.P. Active and semi active vibration isolation systems based on magnetorheological materials. Procedia Eng. 2015, 106, 170-174. [CrossRef] 
5. Ngatu, G.T.; Wereley, N.M.; Karli, J.O.; Bell, R.C. Dimorphic magnetorheological fluids: Exploiting partial substitution of microspheres by nanowires. Smart Mater. Struct. 2008, 17, 045022. [CrossRef]

6. Chen, L.; Gong, X.-L.; Jiang, W.-Q.; Yao, J.-J.; Deng, H.-X.; Li, W.-H. Investigation on magnetorheological elastomers based on natural rubber. J. Mater. Sci. 2007, 42, 5483-5489. [CrossRef]

7. Xuan, S.; Xu, Y.; Liu, T.; Gong, X. Recent progress on the magnetorheological plastomers. Int. J. Smart Nano Mater. 2015, 6, 135-148. [CrossRef]

8. Sun, C.; Pang, H.; Xuan, S.; Gong, X. Glass microspheres strengthened magnetorheological plastomers for sound insulation. Mater. Lett. 2019, 256, 126611. [CrossRef]

9. Liu, T.; Xu, Y.; Gong, X.; Pang, H.; Xuan, S. Magneto-induced normal stress of magnetorheological plastomer. AIP Adv. 2013, 3, 082122. [CrossRef]

10. Xu, Y.; Liu, T.; Wan, Q.; Gong, X.; Xuan, S. The energy dissipation behaviors of magneto-sensitive polymer gel under cyclic shear loading. Mater. Lett. 2015, 158, 406-408. [CrossRef]

11. Gong, X.; Xu, Y.; Xuan, S.; Guo, C.; Zong, L.; Jiang, W. The investigation on the nonlinearity of plasticine-like magnetorheological material under oscillatory shear rheometry. J. Rheol. 2012, 56, 1375-1391.

12. Xu, Y.; Gong, X.; Liu, T.; Xuan, S. Squeeze flow behaviors of magnetorheological plastomers under constant volume. J. Rheol. 2014, 58, 659-679.

13. Lee, S.J.; Kwak, J.W.; Kim, H.D.; Jeon, H.Y.; Kim, J.H.; Yoon, W.S.; Lyoo, W.S. Rheological properties of high molecular weight poly (vinyl alcohol)/dimethyl sulfoxide/water concentrated solutions. Polym. Polym. Compos. 2004, 12, 561-567. [CrossRef]

14. Ahmed, E.M. Hydrogel: Preparation, characterization, and applications: A review. J. Adv. Res. 2015, 6, 105-121. [CrossRef] [PubMed]

15. Mitsumata, T.; Abe, N. Giant and reversible magnetorheology of carrageenan/iron oxide magnetic gels. Smart Mater. Struct. 2011, 20, 124003. [CrossRef]

16. Mitsumata, T.; Sakai, K.; Takimoto, J.I. Giant reduction in dynamic modulus of K-carrageenan magnetic gels. J. Phys. Chem. B 2006, 110, 20217-20223. [CrossRef] [PubMed]

17. Negami, K.; Mitsumata, T. Magnetorheology of magnetic poly(vinyl alcohol) gels with high mechanical toughness. Chem. Lett. 2010, 39, 550-551. [CrossRef]

18. Wu, J.; Gong, X.; Fan, Y.; Xia, H. Physically crosslinked poly(vinyl alcohol) hydrogels with magnetic field controlled modulus. Soft Matter 2011, 7, 6205-6212. [CrossRef]

19. Lawrence, M.B.; Abbas, S.; Aswal, V.K. Structure of polyvinyl alcohol-borax ferrogels: A small angle neutron scattering study. J. Polym. Res. 2018, 25, 36. [CrossRef]

20. Park, G.; McLaurin, E.J.; Faidley, L.E. Characterization of the actuator behavior of blended-system ferrogels. In Proceedings of the Behavior and Mechanics of Multifunctional and Composite Materials 2008, San Diego, CA, USA, 10-13 March 2008; Dapino, M.J., Ounaies, Z., Eds.; Volume 6929, p. 69292C.

21. Akama, S.; Ikeda, J.; Kawai, M.; Mitsumata, T. A feature in magnetorheological effect for polysaccharide magnetic hydrogels. Chem. Lett. 2018, 47, 1240-1242. [CrossRef]

22. Mitsumata, T.; Ohori, S.; Honda, A.; Kawai, M. Magnetism and viscoelasticity of magnetic elastomers with wide range modulation of dynamic modulus. Soft Matter 2013, 9, 904-912. [CrossRef]

23. Kharine, A.; Manohar, S.; Seeton, R.; Kolkman, R.G.M.; Bolt, R.A.; Steenbergen, W.; de Mul, F.F.M. Poly(vinyl alcohol) gels for use as tissue phantoms in photoacoustic mammography. Phys. Med. Biol. 2003, 48, 357-370. [CrossRef]

24. Noorjahan; Pathak, S.; Jain, K.; Pant, R.P. Improved magneto-viscoelasticity of cross-linked PVA hydrogels using magnetic nanoparticles. Colloids Surfaces A Physicochem. Eng. Asp. 2018, 539, 273-279. [CrossRef]

25. Shi, L.; Han, Q. Molecular dynamics study of the influence of solvents on the structure and mechanical properties of poly(vinyl alcohol) gels. J. Mol. Model. 2018, 24, 1-11. [CrossRef] [PubMed]

26. Kudo, S.; Otsuka, E.; Suzuki, A. Swelling behavior of chemically crosslinked PVA gels in mixed solvents. J. Polym. Sci. Part B Polym. Phys. 2010, 48, 1978-1986. [CrossRef]

27. Cha, W.; Hyon, S.; Ikada, Y. Transparent poly (viny1 alcohol) hydrogel with high water content and high strength. Macromol. Chem. Phys. 1992, 193, 1913-1925. [CrossRef]

28. Gupta, D.; Jassal, M.; Agrawal, A.K. The electrospinning behavior of poly(vinyl alcohol) in DMSO-water binary solvent mixtures. RSC Adv. 2016, 6, 102947-102955. [CrossRef] 
29. Ju, B.; Tang, R.; Zhang, D.; Yang, B.; Yu, M.; Liao, C.; Yuan, X.; Zhang, L.; Liu, J. Dynamic mechanical properties of magnetorheological elastomers based on polyurethane matrix. Polym. Compos. 2016, 37, 1587-1595. [CrossRef]

30. Kwon, S.; Lee, J.; Choi, H. Magnetic Particle Filled Elastomeric Hybrid Composites and Their Magnetorheological Response. Materials (Basel) 2018, 11, 1040. [CrossRef]

31. Wang, S.; Jiang, W.W.W.; Jiang, W.W.W.; Ye, F.; Mao, Y.; Xuan, S.; Gong, X. Multifunctional polymer composite with excellent shear stiffening performance and magnetorheological effect. J. Mater. Chem. C 2014, 2, 7133-7140. [CrossRef]

32. Krumova, M.; López, D.; Benavente, R.; Mijangos, C.; Pereña, J. Effect of crosslinking on the mechanical and thermal properties of poly(vinyl alcohol). Polymer (Guildf.) 2000, 41, 9265-9272. [CrossRef]

33. Sorokin, V.V.; Ecker, E.; Stepanov, G.V.; Shamonin, M.; Monkman, G.J.; Kramarenko, Y.; Khokhlov, A.R.; Kramarenko, E.Y.; Khokhlov, A.R. Experimental study of the magnetic field enhanced Payne effect in magnetorheological elastomers. Soft Matter 2014, 10, 8765-8776. [CrossRef] [PubMed]

34. Xu, Y.; Gong, X.; Xuan, S.; Zhang, W.; Fan, Y. A high-performance magnetorheological material: Preparation, characterization and magnetic-mechanic coupling properties. Soft Matter 2011, 7, 5246-5254. [CrossRef]

35. Xu, Y.; Gong, X.; Xuan, S. Soft magnetorheological polymer gels with controllable rheological properties. Smart Mater. Struct. 2013, 22, 075029. [CrossRef]

36. Zhang, G.; Wang, H.; Wang, J.; Zheng, J.; Ouyang, Q. Dynamic rheological properties of polyurethane-based magnetorheological gels studied using oscillation shear tests. RSC Adv. 2019, 9, 10124-10134. [CrossRef]

37. Mitsumata, T.; Ohori, S. Magnetic polyurethane elastomers with wide range modulation of elasticity. Polym. Chem. 2011, 2, 1063. [CrossRef]

38. Jia, Y.; Bai, S.; Park, C.B.; Wang, Q. Effect of Boric Acid on the Foaming Properties and Cell Structure of Poly(vinyl alcohol) Foam Prepared by Supercritical-CO2 Thermoplastic Extrusion Foaming. Ind. Eng. Chem. Res. 2017, 56, 6655-6663. [CrossRef]

(C) 2020 by the authors. Licensee MDPI, Basel, Switzerland. This article is an open access article distributed under the terms and conditions of the Creative Commons Attribution (CC BY) license (http://creativecommons.org/licenses/by/4.0/). 